\title{
O custo do atendimento emergencial às vítimas de violências em dois hospitais do Rio de J aneiro
}

\author{
Emergency care costs for victims of violence \\ treated at two hospitals in Rio de J aneiro
}

Suely Ferreira Deslandes 1

Cosme Marcelo Furtado Passos da Silva 2

Maria Alicia Dominguez U gá 3

\footnotetext{
1 Instituto Fernandes Figueira, Fundação Oswaldo Cruz. Av. Rui Barbosa 716, 4o andar, Rio de Janeiro, RJ 21045-900, Brasil. desland@iff.fiocruz.br

2 Centro Latino-Americano de Estudos deViolência e Saúde Jorge Careli, Fundação Oswaldo Cruz. Av. Brasil 4036, sala 702, Rio de Janeiro, RJ 21040-361, Brasil. passos@manguinhos.ensp. fiocruz.br

3 Escola Nacional de Saúde Pública, Fundação Oswaldo Cruz. Rua Leopoldo Bulhões 1480, sala 709, Rio de Janeiro, RJ 21041-210, Brasil.
}

A bstract The following is a cost analysis of one month of emergency room treatment provided for different types of injuries at two public hospitals in Rio deJaneiro. The study focused on the following: 1) costs at each hospital for treatment of different injuries (annual and monthly estimates); 2) mean cost of each injury; 3) detailed itemized costs of care (materials and drugs, surgery, standard procedures, tests, professional fees, and meals). The items were investigated at the respective hospitals and included services, materials, and resources employed in treating the 1,053 patients included in the sample (498 in the Miguel Couto Municipal Hospital - MCMH and 555 in the Salgado Filho Municipal Hospital - SFMH). Traffic accidents (run-over pedestrians, motor vehicle collisions, and transportation accidents) accounted for $74.3 \%$ of costs at $\mathrm{MCMH}$ and $48.4 \%$ at SFMH. Injuries due to aggression were al so considered rel evant in the cost profile, mainly at SFMH (49\% of costs due to vi olence) as compared to $24.9 \%$ of the total care costs at MCMH. Mean treatment costs for pedestrians run over by motor vehicles varied from $\mathrm{R} \$ 77.76$ (SFMH) to $\mathrm{R} \$ 237.77(\mathrm{MCMH})$. Mean treatment costs for victims of aggression varied from $\mathrm{R} \$ 107.35$ (SFMH) to $\mathrm{R} \$ 84.19(\mathrm{MCMH})$. The study suggests public health measures to deal with and prevent violence. Key words Violence; Hospital Costs; Emergency Medical Services

Resumo O presente trabalho analisa os custos de um mês da assi stência de emergência aos diferentes ti pos de violências atendi dos em dois hospitais municipais do Rio de Janei ro. Este estudo pesquisou: 1) o custo total de cada hospital com o atendimento a cada ti po de violência; 2 ) o custo médio do atendimento por tipo de vi olência; 3) o custo dos atendimentos por seus distintos componentes (materiais e medicamentos, cirurgias, procedimentos padronizados, exames, recursos humanos e alimentação). O estudo se apoi ou em levantamento minucioso in loco de todos os serviços e bens utilizados no atendimento aos 1.053 pacientes da amostra seleci onada (498 no Hospital Miguel Couto - HMMC, e 555 no Hospital Salgado Filho - HMSF). Os aci dentes relacionados ao trânsi to absorveram $74,3 \%$ dos custos da amostra anali sada no HMMC e 48,4\% dos custos relativos à amostra do HMSF. As agressões no HMSF, geraram 49,8\% do custo da vi oIência, ao passo que no HMMC o percentual foi de 24,9\%. O custo médio do tratamento de vítimas dos atropelamentos osci lou entre $\mathrm{R} \$ 77,76$ (HMSF) e $\mathrm{R} \$ 237,77$ (HMMC); os custos médios do aten dimento às víti mas de agressões variaram entre $\mathrm{R} \$ 107,35$ (HMSF) e R\$84,19 (HMMC). A conclusão buscou encaminhar algumas sugestões na perspectiva de reforçar a necessidade de uma agenda na área de saúde pública para o enfrentamento e prevenção da violência.

Palavras-chave Violência; Custos Hospitalares; Serviços Médicos de Emergência 


\section{Introdução}

Nos EUA os custos da atenção às vítimas de violência têm sido um sério problema para os serviços de saúde. Por um lado, por ter aumentado demasiadamente a demanda, pela maior complexidade das lesões ocasionadas pelo uso de armas mais modernas e potentes e pela necessidade de atenção que envolve complexa tecnologia. Por outro lado, por estes custos serem reembolsados apenas parcialmente, cabendo aos fundos públicos arcar com cerca de $70 \%$ a $90 \%$ deste montante (Clancy et al., 1994; Eastman et al., 1991). Tal situação ocasionou o fechamento de 92 centros de trauma entre 1985 e 1991. Discutia-se no Congresso norteamericano a necessidade de se enviar ajuda para custear tais atendimentos. Em Ilinois apresentou-se como proposta a cobrança de uma taxa de US $\$ 20.00$, a ser destinada aos centros de trauma, por cada arma vendida (Skolnick, 1992). Ordog et al. (1995) afirmam que 732 pessoas são baleadas por dia nos EEUU, com um custo médico de um bilhão de dólares ao ano. Para Kizer et al. (1995), estimou-se um custo de quatro bilhões com atenção médica durante o ano de 1995.

Os gastos com atendimento às vítimas de acidentes de trânsito também alcançam estimativas altas. Siegel et al. (1993) apontam um custo americano anual na ordem de 49 bilhões empreendidos com atenção médica, reabilitação e perda de produtividade.

Remenyi et al. (1994) pesquisaram o custo do atendimento de um importante hospital peruano às vítimas de violências. Tomaram como base todas as cirurgias realizadas em 1992 e concluíram que $33 \%$ destas foram devidas a algum tipo de agressão. O gasto com os acidentes atendidos neste hospital equivaleu a U S\$82,902. Baseando-se em estudos anteriores, estes autores estimam um gasto médio de US\$8.88 por atendimento de emergência às vítimas de violências (sem especificar causa).

Waller et al. (1994), caracterizando os custos do atendimento as vítimas de violência em um hospital em Vermont, apontam um custo médio de US\$420.00 para o atendimento emergencial às agressões e U $\$ 2,639$ para o atendimento aos casos de suicídios.

Wintemute \& Wright (1992) estudaram os custos de 250 atendimentos hospital ares causados por lesões por arma de fogo e apontam um custo médio de US\$13,190 por caso (incluindo todo o tratamento).

Em face do crescimento da violência no Brasil, os serviços de saúde têm redirecionado esforços, na tentativa de adequar seus poucos recursos a essa demanda. Na realidade, o impacto dos eventos violentos acarreta um ônus até agora pouco conhecido, o qual se estima ser elevado, porém ainda não quantificado entre nós. Jouvencel (1987) afirma que para cada unidade monetária gasta com prevenção, dez unidades são utilizadas para a assistência e mil para a reabilitação dos casos de violência. DaIossi (1993), pesquisando em um hospital de referência para traumatizados, acompanhou um conjunto de cem casos entre vítimas de acidentes de trânsito, de arma de fogo ou armas brancas, desde a sua internação até a alta ou óbito. Os pacientes vítimas de violência por armas ficaram internados em média 18,7 dias. Números similares foram obtidos para vítimas de acidentes de veículos a motor. Considerando-se que tais casos são potencialmente evitáveis e que estes têm sido demanda crescente nas emergências, percebe-se a importância de conhecer melhor este fenômeno.

Estudo de Ugá (1994) analisou os gastos com internações por violência na região metropolitana do Rio de Janeiro (RMR), no período de 1991 a abril de 1994. A autora contabilizou somente os gastos federais (considerando-se que estes corresponderam a $73 \%$ do total dos gastos neste setor), efetuados na rede hospitalar que compõe o Sistema Único de Saúde - SUS (pública e contratada). Essa análise constatou que há uma tendência crescente, tanto do volume de internações, quanto do número de vítimas. Em 1991 foram gastos US\$1.116.127 para a atenção às vítimas de violência e em 1993 o valor foi de US\$1.891.200 com esta mesma finalidade, havendo, portanto, um aumento de $69 \%$ do gasto hospitalar. As formas de violência intencional (homicídio, suicídio, lesões, acidentes por armas de fogo) foram responsáveis pela maioria absoluta dos gastos com causas externas. O número de internações também variou de forma crescente ano a ano, cumprindo uma média de 2.153 internações por violência ao ano (excluindo-se 1994). Quanto ao tipo de prestador de serviços, verificou-se que as internações efetuadas por hospitais públicos absorveram um volume de recursos 4,5 vezes maior que os privados. Observa-se que mesmo as pessoas que possuem seguros de saúde recorrem ao setor público nas situações de emergências, pois o setor privado, em razão do alto custo destes atendimentos, tem pouca oferta para estes serviços. Percebe-se também que estes valores, embora significativos, não retratam com fidelidade o impacto do problema da violência nos serviços de saúde, uma vez que os valores pagos pelo SUS estão defasados e são repassados com 
considerável atraso. Além disso, este estudo não incluiu os gastos ambulatoriais e aqueles efetuados com internações nas redes estaduais e municipais.

\section{Material e métodos}

\section{Definições}

Este estudo consi derou apenas os custos institucionais do atendimento emergencial da violência, deixando de lado os custos institucionais com internação, reabilitação e aqueles que incidem sobre as vítimas da violência (tangíveis e intangíveis) e sobre a economia como um todo (OPS, 1995). Trata da apuração do valor dos bens e serviços efetivamente utilizados no processo de tratamento desses pacientes (o que é diferente do gasto do hospital, que se refere a um val or desembolsado durante um determinado período na compra de itens não necessariamente utilizados nesse mesmo período).

Nesse sentido, foi necessário identificar as quantidades de cada insumo (tempo de trabaIho profissional, material e medicamentos etc.) utilizado no tratamento de cada paciente de uma amostra (especificada posteriormente). Os valores desses insumos foram expressos em reais $(R \$)$ de maio de 1996. (Nota: no dia 02/ 05/ 96, R\$1,00 equivalia a US\$0,99 - dólar comercial.)

\section{O campo do estudo}

O estudo foi desenvolvido em dois hospitais públicos: Hospital Municipal Miguel Couto (HMMC) e Hospital Municipal Salgado Filho (HMSF). O serviço de emergência do HMMC possui dois andares: o primeiro destina-se às pequenas emergências, dispondo de uma sala para adultos e outra para crianças, sala de ortopedia, sala de otorrinolaringologia e de emergências odontológicas; o segundo andar, destinado às grandes emergências, possui sala de politraumatizados, sala de adultos e outra de crianças (unidade intermediária pediátrica), ortopedia e setor de apoio diagnóstico. $\mathrm{O}$ serviço de emergência do HMSF possui somente um andar com salas de apoio diagnóstico, sala de pequenas emergências (sala de suturas), sala de medicação, sala de politraumatizados (que também atende ali pacientes de UTI), salão de homens e mulheres (que atende tanto trauma, como casos clínicos), duas salas de pediatria e consultório de ortopedia.

A escolha desses hospitais deveu-se ao fato de eles se localizarem em áreas diferenciadas do Município do Rio de Janeiro. O H. M. Miguel Couto situa-se na Zona Sul (a mais rica da cidade) e o H. M. Salgado Filho, na Zona Norte (subúrbio carioca). Apesar de as localizações, geográfica e social, serem bem distintas, estes hospitais não atendem, necessariamente, diferentes demandas no que diz respeito à característica das lesões. Estas diferenças se devem, muito mais, ao perfil de cada hospital (o HMMC é visto como referência para ortopedia e o HMSF é tido como referência para cirurgia, por exemplo). Contudo, por ambos serem hospitais municipais, foram minimizadas as eventuais distinções de âmbito organizacional-administrativo, o que viabilizou uma comparação entre ambos.

Em pesquisa paralela (Deslandes, 1997), investigamos que o $\mathrm{HMMC}$ realizou, em maio de 1996, 19.065 atendimentos de emergência, 2.736 dos quais (14,3\%) deveram-se a violências; o HMSF atendeu em junho do mesmo ano 12.762 casos, sendo 2.415 (18,9\%) também por violências.

\section{Seleção da amostra}

Como seria impossível um registro minucioso de todo o universo de pacientes atendidos por causas externas e pensando-se na qualidade das informações obtidas, adotou-se a técnica de amostragem estratificada, com coeficiente de variação ao nível de 6\%. Esta técnica consiste na subdivisão da população em subpopulações (estratos) que, por sua vez, não se superpõem e, juntos, abrangem toda a população (Cochran, 1965). A partir da determinação dos estratos, selecionou-se uma amostra em cada uma das diferentes subpopulações. Os atendimentos de emergência foram subdivididos em três estratos: 1) agressões, no qual se encontram os casos de agressão física, vítimas de bala perdida e violência doméstica (contra crianças e mulheres). A violência doméstica representa neste caso basicamente a forma de violência física perpetrada por pai ou responsável contra a criança e pelo companheiro ou cônjuge contra a mulher; 2 ) acidentes de trânsito em que se encontram os casos de colisão, atropelamentos e quedas de motos, bem como os acidentes de transporte, como as quedas de ônibus, entre outros; 3) as quedas.

Para o cálculo do tamanho da amostra de cada um dos estratos, foi realizada uma pesquisa prévia dos atendimentos do ano de 1995. Foram escolhidos os meses de maio (HMMC) e junho (HMSF) de 1995 por serem considerados típicos na rotina hospitalar. Assim, levamos em conta toda a população que obteve atendimen- 
to hospitalar de emergência neste período por terem sido vítimas de violências: agressões, acidentes de trânsito, acidentes de transportes e quedas, no mês de maio, no HMMC (1.633 casos); agressões, acidentes de trânsito e de transportes, no mês de junho, no HMSF (545 casos). Com base nestes dados, pudemos definir os tamanhos de amostra de cada um dos estratos a serem coletados em maio e junho de 1996.

Para determinar a variação dos custos dos atendimentos de 1995, e assim cal cular o tamanho da amostra para o estudo de 1996, elegemos uma variável que pudesse retratar esta dimensão. Escolhemos como variáveis o tipo e o número de exames feitos com os pacientes destes diferentes tipos de causas externas. Cabe ressaltar que aferimos a realização destes exames por meio do registro dos boletins e prontuários.

A categoria suicídios não foi incluída no cálculo da amostra, pois, além de possuir grande variabilidade nos custos, foi pequeno o número de casos em maio e junho de 1995. Optou-se por coletar todos os casos desta categoria nos meses de maio (HMMC) e junho (HMSF) de 1996.

Deve-se ressaltar que a categoria queda não participou do cálculo da amostra para o HMSF, uma vez que a categoria acidentes de trânsito e de transportes apresentou grande variabilidade (desvio-padrão) quanto ao tipo e número de exames, o que aumentou, consideravelmente, o tamanho da amostra para este hospital. Assim, precisamos excluir a categoria quedas para possibilitar a execução da pesquisa. Logo, neste hospital consideramos somente as categorias agressões e acidentes de trânsito e de transportes, além da categoria suicídios.

O tamanho de amostra necessário no HMMC para o estrato das agressões seria de 77 e foram coletadas 113; para o estrato dos acidentes de trânsito e de transporte, seriam necessários 150 e foram coletados 190; para as quedas, 125 casos, e foram colhidos 191. No HMSF seriam necessários 105 casos de agressão e o estudo registrou 251; a amostra do estrato dos aci dentes de trânsito e transporte seria de 278 casos e colheu-se 282 . Os casos de suicídio corresponderam a quatro atendimentos no HMMC e a 22 no HMSF. A coleta de dados, portanto, superou o mínimo estipulado no desenho amostral.

\section{Coleta de dados}

O trabalho de coleta de dados dos custos de pacientes vitimados por causas externas foi realizado durante os meses de maio, no HMMC, e junho, no HMSF, no ano de 1996.

Selecionamos e treinamos sete acadêmicos da área de saúde (Medicina e Enfermagem) no último ano do curso de graduação. Estes auxiliares de pesquisa foram dispostos em plantões que se revezavam a cada 12 horas, em esquema de revezamento, durante os 61 dias de pesquisa de campo.

Foi preenchido um formulário específico para a aferição dos custos do atendimento de cada paciente da amostra. A proposta foi a de registrar o mais fidedignamente possível todos os custos do atendimento médico de emergência. O paciente foi acompanhado desde sua admissão no setor de emergência até o término do atendimento, ou por alta médica, transferência para outros setores do próprio hospital fora da emergência, transferência para outro hospital, alta, saída à revelia ou por óbito do paciente.

No decorrer do atendimento médico, os seguintes itens foram registrados por observação direta:

1) Especificação do tipo de profissional envolvido no atendimento. Tempo de atendimento, em minutos, dispensado por estes profissionais em cada procedimento realizado.

2) Materiais, medicamentos e equipamentos utilizados em cada atendimento, sendo todos discriminados criteriosamente segundo especificações de marca, dosagem e quantidade.

3) Tipo de cirurgias executadas.

4) Número de refeições consumidas por cada paciente.

5) Tipo e quantidade de exames de apoio diagnóstico solicitados.

No decorrer do processo de coleta de dados, a demanda de informações a serem registradas pelos pesquisadores - que passou a adquirir um caráter cumulativo, já que muitos pacientes permaneciam em atendimento por mais de 24 horas - tornou-se um desafio à qualidade da informação a ser catalogada. No compromisso de manter o padrão de veracidade dos dados, os pesquisadores foram orientados a notificar os dados registrados nos prontuários ou boletins de atendimento (e confirmá-los junto à equipe de enfermagem do setor) toda vez que fosse impossível observá-los diretamente. Contudo, este expediente foi utilizado poucas vezes, contribuindo para minimizar a perda de informação. 


\section{Codificação}

Para a codificação dos dados obtidos, cada atendimento foi analisado, designando-se uma expressão monetária para cada variável. Os valores monetários foram fornecidos a cada item relacionado segundo as seguintes fontes de informação :

a) Materiais e medicamentos: registros fiscais dos arquivos de entrada do almoxarifado (para os materiais) e da farmácia (para os medicamentos) do HMMC no mês de maio/96, ou mais próximo. Os preços obtidos nos registros fiscais deste hospital foram aplicados para o cálculo nos custos do HMSF.

b) Cirurgias; procedimentos médicos (tais como engessamento, redução de fraturas e luxações); exames diagnósticos: listagem de procedimentos pagos pelo Ministério da Saúde à rede vinculada ao SUS (pública e privada), corrigidos pela taxa de inflação acumulada no período de julho de 1994 a maio de 1996. Efetuou-se este procedimento porque os valores pagos pelo Ministério não foram atualizados desde essa data e, portanto, encontravam-se defasados. Assumiu-se, assim, que os valores definidos em julho de 1994 não correspondiam aos custos atuais e, então, para estimar os custos relativos a maio de 1996, inflacionamos esses valores segundo o Índice Nacional de Preços ao Consumidor (INPC), divulgado pela Fundação Getúlio Vargas. Nesta tabela, já estão contidos os custos de materiais, medicamentos, profissionais e eventual custo de alimentação.

c) Alimentação: tabela de preços para alimentação pagos pela Secretaria Municipal de Saúde - SMS. Por convenção, passamos a contar a diária de alimentação a partir do segundo dia de internação, uma vez que a quase totalidade dos pacientes que recebeu atendimento em menos de 24 horas não utilizou os serviços da nutrição.

d) Recursos humanos envolvidos: calculados com base na relação salário/minuto de trabaIho, de acordo com a fol ha de pagamento dos salários dos profissionais de saúde lotados em emergência fornecida pela SMS, incluindo-se todas as gratificações concedi das aos profissionais. Efetuou-se uma média salarial da classe (média entre os salários dos funcionários em início e fim de carreira). O valor da média da hora trabalhada no HMMC foi ligeiramente maior do que o do HMSF, uma vez que a gratificação de produtividade é maior no primeiro hospital.

A fim de tornar os dados obtidos mais detaIhados, os cál culos de custos foram divididos então em seis grupos: exames; cirurgias; procedimentos médicos padronizados; materiaise medicamentos; profissionais; alimentação.

Classificamos como procedimento médico padronizado os procedimentos ortopédicos envolvendo engessamentos, os quais tiveram seus valores calculados pela listagem de procedimentos médicos pagos na rede SUS multiplicados pelo fator de conversão do INPC. No vaIor final aferido pela tabela SUS, já estariam incluídos desde os recursos humanos utilizados e exames diagnósticos solicitados até o material necessário.

Foram codificados e revisados um total de 1.053 casos de atendimento por causas externas (498 no HMMC e 555 no HMSF). Cada caso foi individualizado em uma tabela de dados visando a sua digitação para um banco D-Base III. Todos os dados foram checados a fim de eliminar possíveis erros de digitação ou tabulação.

\section{Apresentação e discussão dos dados}

Composição do custo total por tipo de violência

Tomando a composição dos custos do tratamento de vítimas da violência da amostra estudada em cada hospital (Tabela 1), observouse que, no HMMC, os atropelamentos absorveram quase a metade $(45,8 \%)$ dos recursos dispensados às causas violentas. Por outro lado, no HMSF as agressões (agressão física, balas perdidas e violência doméstica) geraram a maior parte dos custos da violência, representando $49,8 \%$ do custo total dos tratamentos da vítimas incluídas na amostra.

Deve-se mencionar que era previsível que no HMMC a maior parte dos custos da violência estivesse relacionada a outros acidentes de trânsito e atropelamentos, dado que esse hospital é visto como referência em traumato-ortopedia na rede do SUS. Note-se que, se somadas, essas duas modalidades de violência absorveram nada menos que $67,1 \%$ dos custos da amostra analisada nesse hospital.

Por outro lado, vale notar que, ainda assim, as modalidades agressão física, violência doméstica e balas perdidas, juntas, representaram $24,9 \%$ do custo total dos casos incluídos no estudo nesse mesmo hospital.

No que tange ao HMSF, embora todas as agressões (agressões físicas, violência doméstica, balas perdidas) tenham absorvido a maior parte dos recursos relativos ao tratamento dos casos da amostra (49,8\%), verifica-se que a so- 
Tabela 1

Composição do custo do atendimento emergencial segundo o tipo de violência. HMMC* (maio) e HMSF (junho), 1996.

\begin{tabular}{|c|c|c|c|c|c|c|}
\hline \multirow[t]{2}{*}{ Tipo de violência } & \multicolumn{3}{|c|}{ HMMC } & \multicolumn{3}{|c|}{ HMSF } \\
\hline & $n$ & $\mathrm{R} \$$ & $\%$ & $\mathrm{n}$ & $\mathrm{R} \$$ & $\%$ \\
\hline Agressão física & 90 & $7.576,89$ & 16,7 & 182 & $19.537,39$ & 44,7 \\
\hline Bala perdida & 9 & $3.339,02$ & 7,4 & 9 & 269,26 & 0,6 \\
\hline Violência doméstica & 14 & 339,67 & 0,8 & 60 & $1.971,97$ & 4,5 \\
\hline Suicídio & 4 & 376,70 & 0,8 & 22 & 776,30 & 1,8 \\
\hline Atropelamento & 88 & $20.747,38$ & 45,8 & 128 & $9.952,75$ & 22,8 \\
\hline O utros acidentes de trânsito & 81 & $9.650,99$ & 21,3 & 138 & $10.230,86$ & 23,4 \\
\hline Acidente de transporte & 21 & $3.272,68$ & 7,2 & 16 & 989,12 & 2,2 \\
\hline Total violência & 307 & $45.303,33$ & 100,0 & 555 & $43.727,65$ & 100,0 \\
\hline
\end{tabular}

* Excluíram-se nesta tabela as 191 quedas selecionadas na amostra do HMMC.

ma dos custos de atropelamento e outros acidentes de trânsito representa nada menos que $46,2 \%$ dos custos da amostra estudada neste hospital.

Deve-se observar que, entretanto, pelos motivos referidos na seção "material e métodos", esses dados excluem a modalidade de causa externa mais freqüentemente atendida nesses dois hospitais: as quedas, que constituíram 1.055 dos 2.736 atendimentos realizados em maio no HMM C e 851 dos 2.417 efetuados no HMSF.

Assim, tomando o HMMC, no qual foi possível estudar o custo das quedas, verificou-se que, embora elas tenham representado 38,3\% dos casos amostrados (191), geraram apenas $22,1 \%$ dos custos da violência. Isto se deve ao fato de que o custo médio das quedas foi muito inferior não só ao dos atropelamentos, de outros acidentes de trânsito e de transporte, como também ao dos diferentes tipos de agressões. Incluindo-se as quedas, o comportamento percentual dos custos dos atendimentos no HMMC foi o seguinte: $35,7 \%$ para atropelamentos; $22,1 \%$ para quedas; $16,6 \%$ para outros acidentes de trânsito; $13,0 \%$ para agressão; 5,6\% para acidentes de transporte; 5,7\% para balas perdidas; $0,7 \%$ para suicídios e 0,6\% para violências domésticas.

Os custos médios de cada tipo de violência contidos em nossa amostra podem ser conferidos na Figura 1.

Percebe-se que o custo médio do atendimento de um mesmo tipo de violência variou significativamente de um hospital para outro. Os atendimentos de vítimas de balas perdidas revelam uma diferença de custo da ordem de $1.240,0 \%$; os de atropelamentos, da ordem de $303,2 \%$. Excetuando-se os casos de agressão física e violência doméstica, o custo médio dos atendimentos das violências foi sempre menor no HMSF. Algumas hipóteses podem ser elaboradas. A primeira delas é o fato de o atendimento das vítimas de causas externas ser realizado neste hospital, em significativa parcela (33,9\%), exclusivamente por acadêmicos (Deslandes, 1997).

Ao contrário do que se imagina, o atendimento realizado pelo acadêmico é de baixo custo. Apesar de incluir uma boa parcela de desperdício de material por inexperiência, estes materiais são de baixíssimo custo. Por outro lado, o acadêmico não tem autoridade para solicitar nenhum exame ou realizar qualquer ato cirúrgico de maior complexidade, o que geraria custos mais elevados.

Outra hipótese diz respeito à composição das equipes. Em estudo anterior (Deslandes, 1997), pode-se perceber que no HMSF apenas 7,4\% (176) de todas as vítimas de violência receberam atendimento de mais de um especialista, contra um percentual de $10,5 \%$ (279) no HMMC.

É necessário relativizar tais diferenças, pois o estudo diz respeito a um levantamento referente a um único mês e, portanto, questões como gravidade da lesão, condições de recuperação de cada paciente, condições de funcionamento dos equipamentos de radiologia, disponibilidade de material e composição das equipes podem ter variado de um hospital para outro.

Uma vez considerado cada custo médio dos casos estudados em nossa amostra, pudemos estimar o gasto total em cada hospital para o universo dos atendimentos naquele período. Esta estimativa pode ser construída mediante produto obtido entre o custo médio e o número de todos os casos atendidos segundo o tipo de violência naquele período (referem-se ao total de casos atendidos em cada hospital e não somente à amostra trabalhada). 
Assim, pode-se inferir que o custo dos atendimentos foi o descrito na Tabela 2.

Vale destacar que a estimativa de custo do atendimento das causas externas em ambos os hospitais está subestimada, uma vez que não incorporaram os outros acidentes. No HMSF em especial, os custos estão subestimados, pois não incluíram o cálculo das quedas. Outros acidentes é um caso que corresponde a uma extensa gama de acidentes: domésticos, de trabalho, da prática de esportes, afogamentos, entre outros. Tal variedade de fenômenos impossibilitou sua inclusão neste estudo, já que acarretaria tamanho amostral de impossível exeqüibilidade. Contudo, mesmo que se excluíssem as quedas do HM MC, os gastos deste hospital ainda seria quase o dobro daqueles do HMSF.

Considerando-se o tempo médio dispendido (em horas) no atendimento como um parâmetro auxiliar de análise dos custos, levantouse, em banco de dados de estudo anterior (Deslandes, 1997), que: a) as agressões tiveram um atendimento médio de $8 \mathrm{~h} 3 \mathrm{~min}$ no HMMC e $3 \mathrm{~h}$

\section{Figura 1}

Custo médio (em Reais) do atendimento emergencial segundo tipo de violência. HMMC (maio), HMSF (junho), 1996.

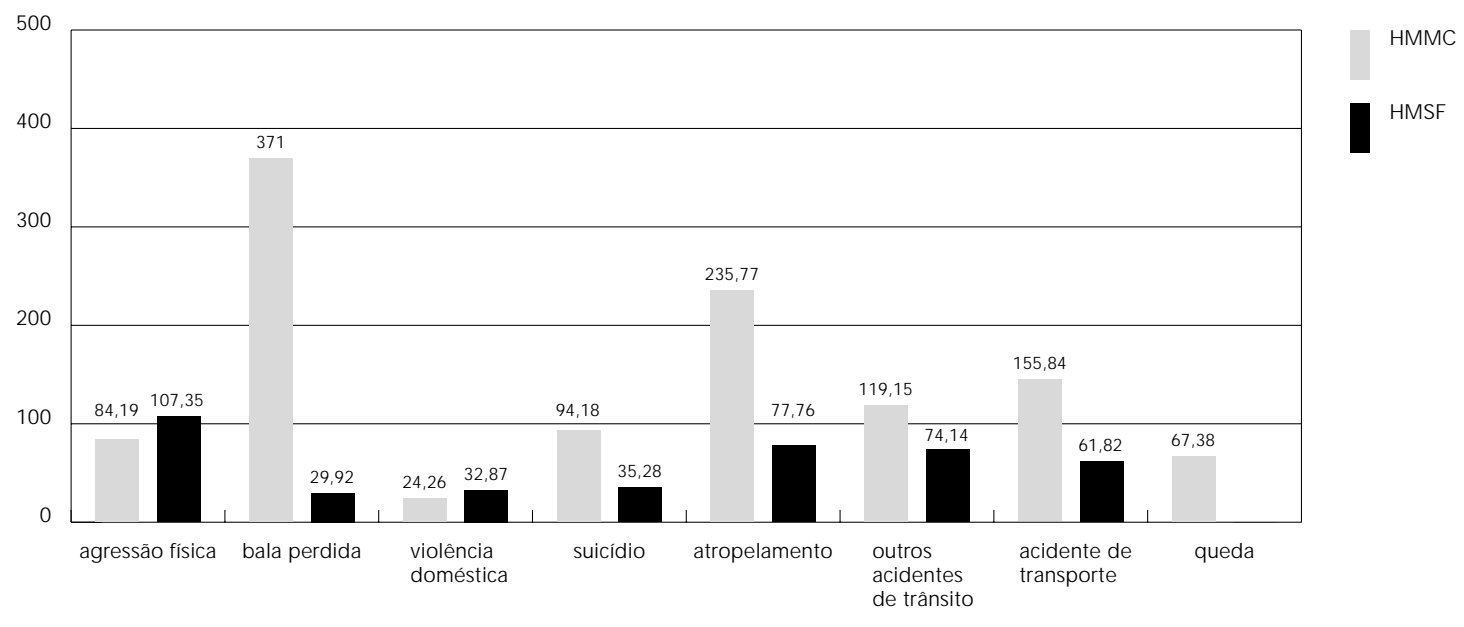

Tabela 2

Estimativa da composição do custo (em Reais) do universo do atendimento emergencial segundo o tipo de violência. HMMC (maio) e HMSF (junho), 1996.

\begin{tabular}{|c|c|c|c|c|c|c|}
\hline Tipo de violência & $\mathrm{n}$ & $\begin{array}{c}\text { HMMC } \\
\text { Custo médio }\end{array}$ & Custo estimado & $\mathrm{n}$ & $\begin{array}{c}\text { HMSF } \\
\text { Custo médio }\end{array}$ & Custo estimado \\
\hline Agressão física & 176 & 84,19 & $14.817,44$ & 188 & 107,35 & $20.181,80$ \\
\hline Bala perdida & 12 & 371,00 & $4.452,00$ & 13 & 29,92 & 388,96 \\
\hline Violência doméstica & 45 & 24,26 & $1.091,70$ & 90 & 32,87 & $2.958,30$ \\
\hline Suicídio & 5 & 94,18 & 470,90 & 22 & 35,28 & 776,16 \\
\hline O utros acidentes de trânsito & 162 & 119,15 & $19.302,30$ & 147 & 74,14 & $10.898,58$ \\
\hline Acidente de transporte & 163 & 155,84 & $25.401,92$ & 132 & 61,82 & $8.160,24$ \\
\hline Queda & 1055 & 67,38 & $71.085,90$ & 851 & S.D. & S.D. \\
\hline Overdose & 32 & S.D. & S.D. & 17 & S.D. & S.D. \\
\hline Outros acidentes & 914 & S.D. & S.D. & 807 & S.D. & S.D. \\
\hline Total Violência & 2.722 & - & $173.873,82$ & 2.410 & - & $54.483,72$ \\
\hline
\end{tabular}

S.D.: sem dados. 
48min no HMSF; b) as vítimas de balas perdidas foram socorridas em média de 10h 41min no HMMC e 10h 18min no HMSF; c) as violências domésticas levaram cerca de $2 \mathrm{~h} 20 \mathrm{~min}$ no HMMC e $2 \mathrm{~h} 32 \mathrm{~min}$. no HMSF; d) os suicídios tiveram $13 \mathrm{~h} 27 \mathrm{~min}$ no HMMC e $17 \mathrm{~h} 26 \mathrm{~min}$ no HMSF; e) os atropelamentos, média de $18 \mathrm{~h}$ 11 min no HMMC e 8 h $26 \mathrm{~min}$ no HMSF; f) os acidentes de trânsito (colisões e outros) levaram tempo médio de atendimento de $11 \mathrm{~h}$ 37 min no HMMC e 7h 51min no HMSF; g) os acidentes de transporte, de $4 \mathrm{~h} 3 \mathrm{~min}$ no HMMC e $2 \mathrm{~h} 32 \mathrm{~min}$ no HMSF; $h$ ) as quedas, de $3 \mathrm{~h}$ e 49min no HMMC.

A estimativa de custos referente ao atendimento de um único mês não possibilita a inferência sobre o comportamento de outros meses. No máximo, aproxima-se à meses mais típicos. Mas, mesmo nestes meses, pequenas diferenças, tais como mais feriados ou mais finais-de-semana, podem corresponder a um aumento da demanda dos casos de violências. Por outro lado, ainda haveria que se considerar o comportamento dos custos nos meses não típicos (dezembro a março e julho) que correspondem a meses de maior demanda das causas externas.

Considerando-se, apenas para efeito de projeção provisória, que o custo de tais atendimentos estudados neste mês correspondam a um comportamento típico em relação ao restante do ano, poder-se-ia aferir que o custo anual do HMMC na atenção a estes grupos de causas externas seria de $\mathrm{R} \$ 2.086 .485,84$.

Persistindo neste exercício (pouco rigoroso do ponto de vista metodológico, porém necessário para se compor um quadro exploratório), pode-se supor que os custos anuais de cada hospital segundo os tipos de violência seriam os descritos na Figura 2.

Estas estimativas indicam que, em apenas dois hospitais públicos do Município do Rio de Janeiro, ter-se-ia, como custo anual do atendimento emergencial às vítimas de acidentes de trânsito (atropelamentos e outros acidentes) e transporte, cerca de $\mathrm{R} \$ 1.345 .612,56$. O custo anual estimado para o atendimento das formas de agressões (agressão física, violência doméstica e bala perdida) seriam, nestes dois hospitais, da ordem de $\mathrm{R} \$ 526.682,40$.

\section{Participação dos atendimentos das causas externas no total de custo hospitalar}

Buscamos aferir, inicialmente, o percentual do custo do tratamento de todas as vítimas de violências atendidas em relação aos custos de cada hospital durante o período estudado.

A aferição dos custos de cada hospital não se encontra especificada por setor. Assim, temse somente o montante de todo o custo de cada hospital e nada se sabe sobre o custo efetivo do setor de emergência. Desta forma, dispunha-se do valor do custo do atendimento emer-

Figura 2

Estimativa do custo anual (em Reais) do atendimento emergencial por tipo de violência. HMMC e HMSF, 1996.

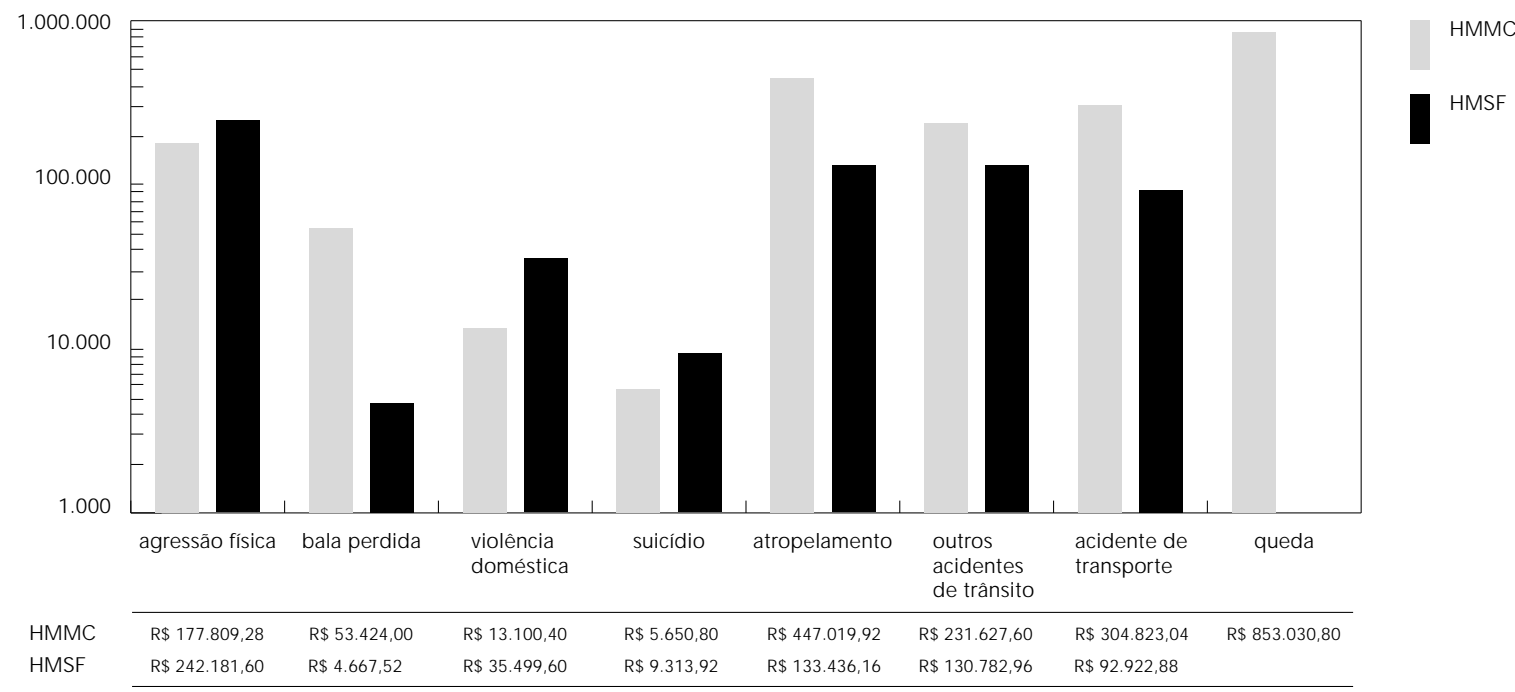


gencial dos casos de violência, mas não havia a base de relevância específica (os custos da emergência).

O cálculo do custo total mensal do atendimento das vítimas da violência em cada hospital foi obtido a partir do produto do custo médio $(\mathrm{Ci})$ de cada tipo de violência pelo número de casos $(\mathrm{Ni})$ atendidos em cada tipo. As porcentagens referidas decorrem da confrontação do somatório dos produtos $\mathrm{Ci}$ x Ni com o custo total mensal do hospital.

Como era de se esperar, a relação percentual ficou diluída numa base excessivamente ampla. O custo do atendimento emergencial das vítimas das diversas formas de violência geraram 7,2\% dos custos do Hospital Municipal Miguel Couto para o mês de maio e 1,9\% dos custos do hospital Municipal Salgado Filho para o mês de junho.

Estas porcentagens são expressivas do ponto de vista qualitativo, levando-se em consideração alguns aspectos. Em primeiro lugar, o porte desses dois hospitais e o elevado número de pacientes por eles atendidos. Em segundo, este estudo referiu-se exclusivamente ao custo do atendimento de emergência. Os custos do atendimento dos pacientes que precisaram ser internados e necessitaram de reabilitação não foram contabilizados. Em terceiro, os dados referentes aos custos de cada hospital dizem respeito ao hospital como um todo e não exclusivamente ao setor de emergência (a Secretaria Municipal ainda está implantando a aferição de custo por setor), logo esta relação percentual seria maior caso a base de cál culo fossem os custos do setor referido. Em quarto lugar, este cálculo só diz respeito aos custos das agressões físicas, violências domésticas, balas perdidas, suicídios, acidentes de trânsito (atropelamentos, colisões e quedas de moto), transportes e quedas, sendo esta última aferida somente no HMMC. Foram excluídos todos os outros tipos de acidentes (914 no HMMC e 807 no HMSF) e as 851 quedas atendidas no HMSF.

Portanto, este percentual é na realidade muito maior do que foi possível e exeqüível de ser aferido por nosso estudo.

O estudo de Remenyi et al. (1994) investigou os custos de 148 atendimentos emergenciais de causas externas que demandaram cirurgias em um grande hospital do Peru (Cayetano Herendia). Seus resultados apontam que estes consumiram $1,1 \%$ do financiamento daquele hospital para o primeiro semestre de 1994. Tais dados corroboram a importância de se dispor de dados mais específicos ao setor de emergência.
Composição do custo total por elemento de custo

Tomando como elementos de custo os exames diagnósticos, as cirurgias, os procedimentos médicos padronizados (gessamentos e imobilizações ortopédicas), os materiais e medicamentos, o tempo de trabalho profissional e os custos de alimentação, observou-se, como era de se esperar, que as cirurgias representaram a maior parte dos custos das violências nos dois hospitais estudados: $47,6 \%$ no HMMC e 52,3\% no HMSF.

Como se observa na Figura 3, os custos da violência foram predominantemente compostos pelos elementos exames e cirurgias, que, juntos, absorveram $70,9 \%$ de tais custos no HMMC e 74,7\% no HMSF.

Também é possível perceber que as cirurgias do HMSF corresponderam a um valor médio mais elevado que as do HMMC. Neste último hospital, 55 pacientes precisaram de cirurgias, correspondendo a um custo médio de $\mathrm{R} \$ 503,56$. No HMSF, 25 pacientes foram submetidos a cirurgia, compondo um custo médio de $R \$ 914,06$.

Dos 498 pacientes da amostra do HMMC, 55 (11\%) fizeram cirurgias, ao passo que, dos 555 pacientes da amostra do HMSF, 25 (4,5\%) submenteram-se a este procedimento.

Quanto a pedido de exames, no HMMC 430 pacientes realizaram algum tipo de exame, enquanto no HMSF este número foi de somente 235 pacientes.

Chama a atenção a baixa participação (3,5\% e $3,9 \%$ ) do custo do trabalho profissional na composição dos custos, refletindo as precárias condições salariais dos profissionais na área de saúde. O valor médio da hora de trabal ho de um médico (média entre o início e o fim de carreira, incluindo todas as gratificações e produtividade) foi de $\mathrm{R} \$ 9,44$ no HMSF e R $\$ 10,26$ no HMMC. A enfermeira teve como valor médio da hora trabalhada $\mathrm{R} \$ 7,01$ e $\mathrm{R} \$ 7,62$, respectivamente no HMSF e no HMMC. O auxiliar de enfermagem recebeu $\mathrm{R} \$ 2,77$ no $\mathrm{HMSF}$ e $\mathrm{R} \$ 3,05$ no HMMC. Tais valores são extremamente baixos dado o desgaste imposto a estes profissionais num setor de emergência.

Outro elemento de custo que chamou a atenção dos pesquisadores pelo seu alto valor unitário foram as refeições pagas em sistema de terceirização. No período da pesquisa, a alimentação para um dia inteiro (cinco refeições) perfaziam o total de $\mathrm{R} \$ 21,63$ e apenas o almoço correspondeu a $\mathrm{R} \$ 6,93$. 
Figura 3

Composição do custo da violência por elemento de custo. HMMC* e HMSF**, 1996.

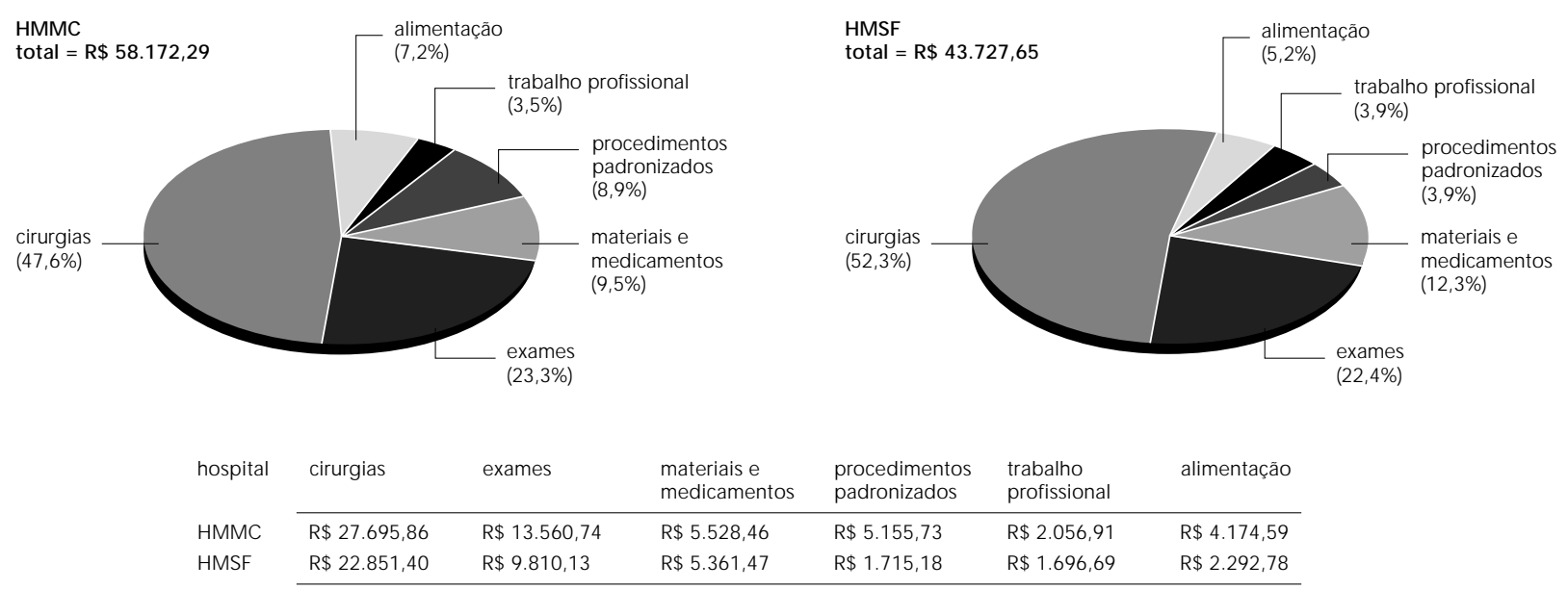

\footnotetext{
* maio/1996
}

** junho/1996

Composição dos custos de cada tipo de violência

A composição dos custos por elemento variou bastante segundo o tipo da violência.

a) No que tange aos suicídios, não houve custo relativo a cirurgias. Predominaram, nos quatro casos do HMMC, os custos referentes a exames, que absorveram $73,4 \%$ dos custos dos suicídios, seguidos dos custos com procedimentos médicos padronizados, que representaram $13,4 \%$ dos mesmos. Já nos 22 casos do HMSF, a maioria dos custos (70,2\%) correspondeu aos materiais e medicamentos. Tais diferenças podem ser compreendidas à luz das diferentes lesões provocadas pelos distintos meios utilizados para a tentativa de suicídio.

b) No HMMC, os 21 casos de acidentes de transporte ocasionaram custos predominantemente referentes a cirurgias (46,5\%). Ressaltese que foram apenas dois casos que precisaram de intervenção cirúrgica. Os custos de materiais e medicamentos corresponderam a 16,9\%; os de exames, a $13,4 \%$, e procedimentos padronizados, a 10,6\%. Nos 16 casos do HMSF, os exames superaram as cirurgias em termos de custo, absorvendo respectivamente $42,0 \%$ e $36,4 \%$.

c) Os atropelamentos e os outros acidentes de trânsito apresentaram uma composição de custos semelhante: predominaram, no HMMC, os custos relativos às cirurgias (representando $47,4 \%$ e $48,7 \%$ respectivamente) e, em segundo lugar, destacaram-se os custos relativos a exames, que absorveram $25,5 \%$ e $27,5 \%$ dos custos de cada um desses tipos de violência naquele hospital. O terceiro item de custo foi alimentação $(11,1 \%)$, para os casos de atropelamentos, e materiais e medicamentos (7,9\%), para os casos de outros acidentes de trânsito. No HMSF, observou-se nos casos de atropelamentos o predomínio do custo com cirurgias como primeiro item (36,6\%), os custos de exames como segundo item $(32,2 \%)$ e os custos com materiais e medicamento (10,5\%) como terceiro. Nos casos de outros acidentes de trânsito, o primeiro lugar dos custos foram os exames $(35,7 \%)$, o segundo foram as cirurgias $(31,4 \%)$ e o terceiro foram os materiais e medicamentos (15,9\%).

d) A violência doméstica não gerou nenhuma cirurgia nos 14 casos do HMMC Predominaram nestes os custos com procedimentos médicos padronizados, que absorveram $47,5 \%$ dos custos desse tipo de violência; destacaram-se, em segundo lugar, os custos com exames (30,3\%). Situação inversa se observou nos sessenta casos do HMSF, no qual as duas únicas cirurgias representaram o principal elemento do custo da violência (78,8\%).

e) As modalidades agressões físicas e balas perdidas concentraram seus custos no elemento cirurgia, que, no HMMC, absorveu respectivamente $51,5 \%$ e $86,1 \%$ dos custos. O segundo elemento de custo, em ordem de importância, nesse mesmo hospital, foi o relativo a exames, 
que representou $21,7 \%$ do custo das agressões e $5,8 \%$ do relativo às balas perdidas. Assim como no HMMC, os elementos de custo que tiveram uma mai or concentração para a modalidade agressões físicas no HMSF foram as cirurgias ( $72,2 \%$ ) e os exames ( $11,6 \%)$, o mesmo não acontecendo com a modalidade balas perdidas, cuja concentração foram os materiais e medicamentos (34,3\%), seguindo-se pelo tempo dedicado pelos profissionais às vítimas (20,4\%).

f) As 191 quedas atendidas no HMMC tiveram um custo distribuído fundamentalmente entre as cirurgias (37,9\%), os procedimentos padronizados (24,3\%) e os exames (23\%).

\section{Considerações finais}

As considerações finais necessitam ser precedidas de algumas questões que consideramos fundamentais para a compreensão da contribuição deste estudo e de suas limitações.

A dimensão dos custos das violências é bastante ampla e este estudo investigou somente uma parcela desta totalidade. Trabalhou-se com um tipo específico de custos diretos das violências: o atendimento assistencial de emergência. Assim, todos os outros custos diretos ligados à assistência, como o atendimento hospitalar e o tratamento de reabilitação não foram considerados. Desconsideramos ainda os custos pessoais e familiares com o tratamento e reabilitação das vítimas. Também não foram incluídos os custos indiretos, como a perda de produtividade e as perdas materiais decorrentes dos agravos.

Este estudo ainda se revela como exploratório, uma vez que pouco se investigou sobre tal temática no país e, portanto, as metodologias e estratégias de investigação ainda se afirmam. Recorreu-se, portanto, a diferentes fontes de aferição de custos que representam lógicas diferentes (notas de compra de almoxarifado dos hospitais para os materiais e medicamentos; tabelas do SUS para os procedimentos cirúrgicos e procedimentos médicos padronizados; cál culo direto do valor da hora dos profissionais).

Este exercício, contudo, não resolve a defasagem dos valores pagos pelo SUS à rede hospitalar municipal; na verdade, trabalhou-se com valores extremamente defasados. Entretanto, esta realidade diz respeito não somente ao atendimento às vítimas de violência, mas a todo atendimento realizado na rede pública.

Por outro lado, as fontes de registro hospitalar quanto ao tipo de causa externa são mui- to precárias, o que inviabiliza a determinação de amostras adequadas. Assim, ainda que num único mês, não foi possível estudar todos os tipos de causas externas. Excluíram-se, por exemplo, as quedas da amostra do Salgado FiIho e todos os outros acidentes dos dois hospitais. Desta forma, obteve-se uma estimativa mensal dos custos emergenciais do atendimento ainda sub-registrada.

Outra limitação importante diz respeito à impossibilidade de se verificar o percentual dos custos dos atendimentos às vítimas de violências em relação ao total de custos do serviço de emergência. Tal impedimento se deve à não-existência dos dados de custos por setor de cada hospital. Os dados disponíveis na Secretaria Municipal de Saúde referem-se à totalidade de custos do hospital como um todo. O sistema de aferição por setor está em processo de implantação.

Ainda que com tais limitações, o presente estudo traz importantes contribuições quando aponta um custo médio do atendimento emergencial por cada tipo de violência e uma estimativa mensal deste custo. Foi possível, portanto, construir um mapa em linhas amplas de um problema grave e pouco explorado.

Da análise apresentada acima, depreendese que, nos hospitais estudados, os acidentes relacionados ao trânsito (atropelamentos, acidentes de trânsito e acidentes de transporte) absorveram a maior parte dos custos do tratamento de vítimas de violência: juntas, estas três modalidades representaram nada menos que $74,3 \%$ dos custos da amostra anal isada no $\mathrm{HMMC}$ e 48,4\% dos relativos àquela do HMSF, quando excluído o item queda.

No HMMC, em que foi possível estudar o custo das quedas da amostra, verificou-se que estas representaram $22,1 \%$ dos custos da violência e eram a maior parte dos casos (38,3\%). Ao incluí-las no cálculo total dos custos da violência neste hospital, os três itens relacionados ao trânsito passaram a representar 57,9\% desse total.

Por outro lado, as agressões constituíram também um importante fator de custo, principalmente no HMSF. Elas geraram nada menos que $49,8 \%$ do custo da violência nesse hospital.

Evidenciou-se, também, ao longo da análise, a heterogeneidade dos custos médios dos diversos tipos de violência: eles variaram de $\mathrm{R} \$ 24,26$ (violência doméstica) a $\mathrm{R} \$ 371,00$ (balas perdidas) no HMMC e de $\mathrm{R} \$ 29,92$ (balas perdidas) a R\$107,35 (agressão) no HMSF.

Vale mencionar também a variação dos custos observados nos dois hospitais, embora 
sejam ambos da rede municipal: o custo médio do tratamento de vítimas dos atropelamentos oscilou entre $\mathrm{R} \$ 77,76$ (HMSF) e R $\$ 237,77$ (HMMC). No que tange aos acidentes de transportes, seu custo médio variou entre $R \$ 61,82$ (HMSF) e R $\$ 155,84$ (HMMC).

A variação dos custos apresentada pelos dois hospitais se verificou também em relação à comparação dos custos de cada tipo de violência: enquanto no HMMC as balas perdidas ocasionaram os tratamentos de maior custo médio ( $R \$ 371,00)$, sendo seguidas pelos atropelamentos $(\mathrm{R} \$ 235,76)$, no HMSF as agressões geraram os tratamentos de maior custo médio $(R \$ 107,35)$, sendo seguidas pelos acidentes de trânsito $(\mathrm{R} \$ 74,14)$.

De um lado, a variação dos custos entre os distintos tipos de violências era esperada, uma vez que provocam lesões de ordens distintas (por exemplo, uma briga gera lesões diferentes de um atropelamento).

Por outro lado, a variação de custo em relação ao mesmo tipo de violência, em dois hospitais que, teoricamente, deveriam prestar o mesmo padrão de socorro, obrigam a iniciar uma reflexão sobre o assunto. Podem-se elaborar, provisoriamente, al gumas hipóteses explicativas:

- Composição diferenciada das equipes médicas: ter ou não determinado especialista nos plantões; envolver ou não o atendimento de um único ou vários especialistas conduzem a um padrão diferenciado de atendimento e, portanto, de custos.

- Participação majoritária do acadêmico no atendimento como fator de minimização dos custos.

- Possível existência de rotinas diferenciadas de atendimento em cada um dos hospitais.

- Variação de recursos diagnósticos disponíveis em cada hospital.

- Variação da gravidade das lesões nas amostras selecionadas.

Finalmente, deve-se observar que, como era previsível, as cirurgias foram o elemento de custo mais importante quando considerado o custo total de todas as vítimas da violência na amostra de cada hospital. Este procedimento absorveu nada menos que $47,6 \%$ e $52,3 \%$ dos recursos alocados ao atendimento das vítimas, respectivamente, pelos hospitais Miguel Couto e Salgado Filho. Seguiram, em ordem de importância, os custos relativos a exames, que representaram $23,3 \%$ no HMMC e $22,4 \%$ no HMSF. Em terceiro lugar, estão os materiais e medicamentos, que absorveram respectivamente $9,5 \%$ e $12,3 \%$ dos custos da violência realizados no $\mathrm{HMMC}$ e no HMSF.
Uma menção final deve ser feita em relação à baixa participação dos salários na composição dos custos da violência: ela é reflexo da vergonhosa defasagem salarial a que estão submetidos os trabalhadores do setor da saúde. Por outro lado, percebeu-se um valor de refeições bem alto, quase o equivalente ao preço de mercado.

É necessário destacar, para concluir, que os resultados deste estudo, que pretendeu apurar com maior nível de exatidão possível os custos da violência, constituem, na realidade, apenas uma estimativa desses custos.

Contudo, os dados deste estudo de custos podem indicar inicialmente alguns pontos para desdobramento de ação futura.

Em relação aos casos de violências:

1) Premência de campanhas que auxiliem na prevenção das violências, pois estas constituem um custo significativo para a assistência hospitalar. Tal questão é especialmente relevante no que diz respeito aos acidentes de transporte/ trânsito, já que estes representaram $74,3 \%$ dos custos da amostra do HMMC e 48,4\% do HMSF, e sabe-se que correspondem a um tipo de violência consideravelmente suscetível às campanhas de prevenção.

2) Necessidade de reflexão sobre a existência de patamares muito distintos de custos do atendimento para o mesmo tipo de violência nos dois hospitais. Estariam refletindo padrões de atendimento diferenciados?

3) Importância de se estimularem novas pesquisas neste tema, consolidando referencial metodológico e apurando mais minuciosamente os custos da assistência de saúde aos distintos tipos de violências.

Em termos gerais:

1) Relevância e premência de se implantar o sistema de aferição de custos por setor, já em andamento na Secretaria Municipal de Saúde. 2) Urgência de implementação de uma política que corrija os aviltantes valores correspondentes ao trabalho profissional.

3) Urgência de consolidação de mecanismos que obriguem o ressarcimento por parte dos seguros privados de saúde ao setor público pelo atendimento realizado em seus consorciados. 


\section{Agradecimentos}

Pesquisa realizada com o apoio da Secretaria Municipal de Saúde do Rio de Janeiro.

\section{Referências}

CLANCY, T. V.; MISICK, L. N.; COVINGTON, D.; CHURCHILL, M. P. \& MAXWELL, J. G., 1994. The financial impact of intentional violence on community hospitals. Journal of Trauma, 37:1-3.

COCHRAN, W. G., 1965. Técnicas de Amostragem. Rio de Janeiro: Fundo de Cultura.

DALOSSI, T., 1993. Determinação Precoce do Nível de Gravidade do Trauma. Dissertação de Mestrado, São Paulo: Escola de Enfermagem, Universidade de São Paulo.

DESLANDES, S. F., 1997. O Impacto da Violência na Emergência Hospitalar. Rio de Janeiro: Centro Latino Americano de Estudos de Violência e Saúde "Jorge Careli", Escola Nacional de Saúde Pública, Fundação Oswaldo Cruz. Relatório Final de Pesquisa. (mimeo.)

EASTMAN, A. B.; RICE, C. L.; BISHOP, G. S. \& RICHARDSON, J. D., 1991. An analysis of the critical problem of trauma center reimbursement. Journal of Trauma, 31:920-926.

JOUVENCEL, M. R., 1987. Salud, Educacion y Violencia. Madrid: Ed. G. J. Knapp.

KIZER, K. W.; VASSER, M. J.; HARRY, R. L. \& LAYTON, K. D., 1995. Hospitalization charges, costs, and income for firearm-related injuries at a university trauma center. Journal of the American Medical Association, 273:1.768-1.773.

ORDOG, G. J.; WASSERBERGER, J. \& ACKROYD, G., 1995. Hospital costs of firearm injuries. The Journal of Trauma, 38:291-298.

OPS (Organização Panamericana de Saúde), 1995. Re comendaciones para el Estudio de los Costos de la Violencia. Caracas: OPS. (mimeo.)
REMENYI, M. A.; CHANG, J. \& BACA, L., 1994. Analisis del Impacto Economico de la Violencia y Negligencia sobre las Instituiciones de Salud. Estudio de Caso (Peru). Lima: OPS (Organização Panamericana de Saúde) - OMS (Organização Mundial de Saúde). Relatório Final de Pesquisa. (mimeo.)

SIEGEL, J. H.; MASON-GONZALES, S.; DISCHINGER, P. C.; READ, M.; CUSHING, B. M.; BADELLINO, M. C.; GOODARZI, S.; SMIALEK, J. E.; HEATFIELD, B. M.; ROBINSON, R. M.; PARKINSON, K.; HILL, W. J.; JACKSON, J. W.; LIVINGSTON, D. J.; BENTS, F. D. \& CLARK, C. C., 1993. Causes and costs of injuries in multiple trauma patients requiring extrication from motor vehicle crashes. Journal of Trauma, 35:920-931.

SKOLNICK, A. A., 1992. Congress acts to resuscitate nation's financially ailing trauma care systems. Journal of the American Medical Association, 267: 2.994-2.995.

UGÁ, M. A. D., 1994. Impacto Económico dela Violencia sobre los Servicios deSalud en Pai ses deAmérica Latina y el Caribe: El Caso de Rio deJaneiro. Rio de Janeiro: Escola Nacional de Saúde Pública, Fundação Oswaldo Cruz. (mimeo.)

WALLER, J. A.; SKELLY, J. M. \& DAVIS, J. H., 1994. Characteristics, costs, and effects of violence in vermont. Journal of Trauma, 37:921-927.

WINTEMUTE, G. J. \&WRIGHT, M. A., 1992. Initial and subsequent costs of firearm injuries. Journal of Trauma, 33:556-560. 